\title{
Immunological and C-peptide studies of patients with diabetes in northern Ethiopia: existence of an unusual subgroup possibly related to malnutrition
}

\author{
G. V. Gill • A. Tekle • A. Reja $\cdot$ D. Wile $\cdot$ P. J. English • \\ M. Diver • A. J. K. Williams $\cdot$ S. Tesfaye
}

Received: 23 April 2010 /Accepted: 20 August 2010 /Published online: 2 October 2010

(C) Springer-Verlag 2010

\begin{abstract}
Aims/hypothesis Surveys in northern Ethiopia have demonstrated that apparent type 1 diabetes occurs more frequently than elsewhere in Africa and, indeed, in other parts of the world. We therefore investigated in detail a cohort of diabetic patients from this region to clarify the nature of this type of diabetes.

Methods All patients attending the diabetic clinic at Mekelle Hospital in the Tigray region of northern Ethiopia were investigated over a 6 week period. Clinical, demographic and anthropometric data were collected, as well as measurements of $\mathrm{HbA}_{1 \mathrm{c}}$, fasting lipid profile, fasting serum Cpeptide and serum markers of beta cell autoimmunity, i.e. islet antigen-2 and GAD antibodies (GADA).

Results Of 105 patients seen, $69(66 \%)$ were on insulin treatment and had been from or close to diagnosis. Their median age and diabetes duration were 30 and 5 years,
\end{abstract}

\section{G. V. Gill $(\bowtie)$}

Department of Tropical Medicine,

Liverpool School of Tropical Medicine,

Liverpool L3 5QA, UK

e-mail: g.gill@liv.ac.uk

A. Tekle

Department of Medicine, Mekelle Hospital,

Mekelle, Ethiopia

\section{A. Reja}

Department of Diabetes/Endocrinology,

Black Lion Hospital,

Addis Ababa, Ethiopia

D. Wile

Department of Clinical Biochemistry,

Aintree University Hospital,

Liverpool, UK respectively, with a male excess of 2:1. Median BMI was $20.6 \mathrm{~kg} / \mathrm{m}^{2}$. Despite these clinical characteristics suggestive of type 1 diabetes, only 42 of 69 (61\%) patients were Cpeptide-negative and 35\% GADA-positive. Overall, 38 $(36 \%)$ of the total group $(n=105)$ had immunological or C-peptide characteristics inconsistent with typical type 1 or type 2 diabetes. The clinical characteristics, local prevalence of undernutrition, and GADA and C-peptide heterogeneity suggest a malnutrition-related form of diabetes. Conclusions/interpretation Not all patients in northern Ethiopia with apparent type 1 diabetes appear to have the form of disease seen in Europids; their disease may, in fact, be related to malnutrition.

Keywords Atypical diabetes mellitus · C-peptide - Diabetes mellitus - GAD antibodies $\cdot$ Malnutrition-related diabetes mellitus - Type 1 diabetes Type 2 diabetes

G. V. Gill • P. J. English

Department of Diabetes/Endocrinology,

Aintree University Hospital,

Liverpool, UK

M. Diver

Department of Clinical Biochemistry,

Royal Liverpool University Hospital,

Liverpool, UK

\section{A. J. K. Williams}

Diabetes and Metabolism,

School of Clinical Sciences,

University of Bristol,

Bristol, UK

\section{S. Tesfaye}

Department of Diabetes/Endocrinology,

Royal Hallamshire Hospital,

Sheffield, UK 


\section{Abbreviations \\ GADA GAD antibodies \\ IA-2 Islet antigen 2 \\ IA-2A IA-2 antibodies}

\section{Introduction}

The provision of diabetes care in Africa, the world's poorest continent, is a major challenge. Inadequate resources and shortage of insulin are a constant barrier to effective care delivery [1]. In addition, patient characteristics sometimes challenge standard classifications of diabetes [2]. An example is 'malnutrition-related diabetes mellitus', a form of diabetes that is characterised by varying degrees of insulin dependence and is often reported in young men from localised geographical areas, sometimes accompanied by pancreatic calcification [3]. More recently, an 'atypical type 2' syndrome has been described in Africa, where initial insulin-dependence may later remit [4]. A viral aetiology for this type of diabetes has been suggested [5].

For some time, unusual phenotypes of diabetes have been observed in northern Ethiopia, but have never been intensively investigated. In the central capital city (Addis Ababa) and surroundings, Lester reported childhood diabetes to be 'typical type 1 ' and 'clinically similar to elsewhere' [6]. Similarly, standard type 2 diabetes, often accompanied by being overweight, is also seen in this area $[7,8]$. However, in the remote and poor northern areas most diabetic patients seen are young, thin and apparently have type 1 diabetes. This has been reported from Mekelle [9] and also from Gondar $[10,11]$, with an even higher proportion in rural areas [11]. Although no accurate incidence figures exist, this frequency of type 1 diabetes has been described as 'astonishing' [11] and is in marked contrast to observations from sub-Saharan Africa, where the vast majority of diabetic patients have type 2 disease $[2,12]$. Interestingly, however, these young and thin northern Ethiopian diabetic patients rarely, if ever, suffer diabetic ketoacidosis and frequently survive despite absent supplies of insulin [9]. A recent survey from rural Ethiopia again confirmed the existence of unusually large numbers of young (peak age 25 to 29 years) insulin-treated patients ( $45 \%$ of the diabetic population of the clinic) with low BMI [13]. Most of these patients were poor subsistence farmers or were unemployed.

The phenotype of apparent type 1 diabetes in this area therefore appears unusual. Several authors have suggested a direct link with malnutrition [9, 13], particularly as famine is common in the area. We therefore undertook a detailed clinical, biochemical and immunological study of a group of these patients from Mekelle, northern Ethiopia.

\section{Methods}

Location The research site was the diabetic clinic, held weekly at Mekelle Hospital in Mekelle, in the Tigray region of northern Ethiopia. Mekelle has a population of 200,000 and has the central referral hospital for Tigray region, which has a population of about 4 million. Much of Tigray is semi-desert, with low rainfall and difficult unsurfaced roads. Most inhabitants are subsistence farmers with very low income. Serious food shortages due to crop failures are common, and particularly severe famines occurred in the mid and late 1980s. Transport is difficult and patients often have to travel large distances on foot (often 50 to $100 \mathrm{~km}$ or more) for medical services. The study was not carried out in the rainy season, when patients from rural areas find it especially difficult to reach the hospital due to impassable roads and tracks. Even when patients do reach hospital, essential drugs and equipment (including insulin and syringes) may be unobtainable.

Patients The diabetic clinic at Mekelle Hospital is the only care facility for diabetic patients in the area. Consecutively attending patients at the diabetic clinic were studied over a 6 week period, amounting to 105 patients. Patients were diagnosed by WHO criteria [14], using a portable blood glucose meter. The type of meter and quality control systems available varied according to supply. In practice, most patients originally presented with obvious osmotic symptoms and marked hyperglycaemia. A written proforma was filled in for each patient recording name, age, location of residence, duration of diabetes, family history of diabetes, treatment, known complications and other illnesses. Patients were divided into 'insulin-treated' and 'non-insulin treated'. The decision to treat with insulin was made by one clinician (A. Tekle), an experienced provider of diabetes care in the area. Each patient was later given a code number and anonymised data were entered on to an Excel computer spreadsheet.

Anthropometric data BMI was determined by dividing weight in $\mathrm{kg}$ by height in metres squared. Weight was measured in light clothes on standard scales to the nearest $0.1 \mathrm{~kg}$. Height was measured to the nearest centimetre using a wall-mounted measuring rod. Blood pressure was measured with a standard mercury sphygmomanometer with the patient in a sitting position and the arm at midthoracic level. Measurement was made to the nearest $\mathrm{mmHg}$ and the diastolic level was recorded as complete disappearance of sounds.

Laboratory data No hospital-based laboratory services (including blood glucose and $\mathrm{HbA}_{1 \mathrm{c}}$ ) were available. Patients attended the clinic in a fasted state and blood was 
taken into plain tubes. A finger prick blood sample was used to measure $\mathrm{HbA}_{1 \mathrm{c}}$ with a portable analyser (Glycosal; Provalis Diagnostics, Queensferry, UK) provided for the study. This system uses boronate affinity chromatography and is aligned to Diabetes Control and Complications Trial criteria [15]. The technique has been shown to work well in tropical field conditions [16]. Blood taken into the plain tube was allowed to clot and the serum was separated by centrifugation at $2,000 \mathrm{~g}$ and stored frozen at $-40^{\circ} \mathrm{C}$. Later, one of the researchers transported the samples on dry ice to the UK. The samples arrived fully frozen and in good condition. Cholesterol, HDL-cholesterol and triacylglycerol were measured by standard laboratory methods on an automated analyser (Hitachi 747; Hitachi, Bohemia, NY, USA) at the Aintree University Hospital, Liverpool, UK. LDL-cholesterol was calculated by the Friedewald equation. C-peptide was measured by radioimmunoassay at the Royal Liverpool University Hospital, Liverpool (an accredited national centre for this assay). The assay is WHO-standardised, with undetectability defined as a level of $<183 \mathrm{pmol} / \mathrm{l}$. The intra-assay coefficient of variability was $1.3 \%$ and precision was $1.8 \%$. Patients with undetectable C-peptide were considered 'C-peptide-negative' and all others 'C-peptide-positive'. The latter group had levels ranging from 225 to $>7,282 \mathrm{pmol} / 1$, but $80 \%$ had values over $400 \mathrm{pmol} / \mathrm{l}$. GAD antibodies (GADA) and islet antigen 2 (IA-2) antibodies were measured at the Department of Diabetes and Metabolism, Southmead Hospital, University of Bristol, UK, as previously described $[17,18]$ and using ${ }^{35}$ S-labelled full-length GAD65 or IA-2 (605-979). Results were expressed in WHO units $/ \mathrm{ml}$ derived from a standard curve and samples were considered positive if above the 97.5th centile (14.0 WHO units/ml for GADA, 6.0 WHO units/ml for IA-2 antibodies [IA-2A]). The GADA and IA$2 \mathrm{~A}$ assays achieved a laboratory-defined sensitivity of $94 \%$ and $69 \%$, with $96 \%$ and $98 \%$ specificity, respectively in the Fourth Diabetes Antibody Standardisation Program [19].

Statistics Statistical evaluation was done using a StatsDirect package. Mean and standard deviations were calculated for normally distributed data; for skewed data, medians with inter-quartile ranges were calculated. Groups were compared for significance by Student's unpaired $t$ test (normal data) or the Mann-Whitney $U$ test (non-normal data). Fisher's exact test was used to compare proportionate variables. The Kruskal-Wallis test was used for comparison between groups. A value of $p<0.05$ was taken to indicate statistical significance.

Ethics We obtained approval for the project from the Ethics Committee of the Mekelle Health Board. Due to the high level of illiteracy, verbal consent only was obtained from patients, who were universally enthusiastic to take part, as for the first time they were able to have detailed biochemical assessment of their diabetes $\left(\mathrm{HbA}_{1 \mathrm{c}}\right.$, lipids, etc.).

\section{Results}

Patient details Of the 105 patients, 74 (68\%) were men, mean $( \pm \mathrm{SD})$ age was $41 \pm 16$ years and diabetes duration (median [interquartile range]) was $5(3-10)$ years. A family history of diabetes was present in ten $(9 \%)$ patients; median (interquartile range) BMI was 20.6 (18.5-23.9). Treatment was with diet $(n=1)$, oral agents $(n=35)$ and insulin $(n=69)$. Only $5 \%$ of patients were hypertensive (BP $>140 / 80$ ). Glycaemic control was poor with $\mathrm{HbA}_{1 \mathrm{c}}$ high at $11.3 \pm 2.8 \%$.

Comparison of insulin-treated and non-insulin treated patients As shown in Table 1, two-thirds of the group were on insulin treatment. They were significantly younger, although of similar diabetes duration to those on oral agents and diet. BMI and both systolic and diastolic BP were also highly significantly lower in those on insulin than in those on other treatments, but glycaemic control was worse $\left(\mathrm{HbA}_{1 \mathrm{c}} 11.9\right.$ vs $\left.10.0 \%, p=0.002\right)$, The lipid profiles were better in the insulin-treated group, with lower total cholesterol and LDL-cholesterol, higher HDL-cholesterol and lower triacylglycerol (all statistically significant except for HDL-cholesterol). Although all these characteristics suggest that the group studied had predominantly or entirely type 1 diabetes, only $35 \%$ were either GADA- or IA-2A-positive. In addition, although C-peptide levels were lower and significantly more patients were C-peptidenegative, $39 \%$ of those on insulin still had detectable levels of fasting serum C-peptide.

Analysis of GADA and C-peptide status Analysis showed that $29(28 \%)$ patients were GADA-positive and $45(43 \%)$ were C-peptide-negative. Only four patients were IA-2Apositive, two of whom were also GADA-positive. Table 2 divides the total group into patients who were GADApositive or -negative, and those who were C-peptide-negative or -positive. Most (83\%) but not all GADA-positive patients were on insulin, and only $62 \%$ were C-peptide-negative. Similarly, some (7\%) C-peptide-negative patients were not on insulin and only $40 \%$ were GADA-positive. The C-peptidepositive and GADA-negative groups showed similar heterogeneity. This led us to arbitrarily group the patients into: (1) 'definite' type 1 diabetes (defined as GADA-positive, Cpeptide-negative and on insulin treatment); (2) 'definite' type 2 diabetes (defined as GADA-negative, C-peptide-positive and on any treatment); and (3) an 'uncertain' group (Table 3). This 'uncertain' group contained $38(36 \%)$ patients. They were of similar age, diabetes duration, $\mathrm{BMI}$ and $\mathrm{HbA}_{1 \mathrm{c}}$ as the type 1 group. In addition, four (11\%) were not on 
Table 1 Comparison of insulinand non-insulin-treated diabetic patients in northern Ethiopia

Unless otherwise indicated, data are means \pm SD for normally distributed data and median (interquartile ranges) for nonnormally distributed data

\begin{tabular}{|c|c|c|c|}
\hline Characteristic & Insulin-treated & Non-insulin treated & $p$ value \\
\hline$n(\%)$ & $69(66)$ & $36(34)$ & \\
\hline Age (years) & $30(22-48)$ & $50(40-60)$ & 0.0001 \\
\hline Duration diabetes (years) & $5(3-10)$ & $6(2-11)$ & NS \\
\hline BMI $\left(\mathrm{kg} / \mathrm{m}^{2}\right)$ & $19.3(16.6-21.3)$ & $24.0(21.2-25.7)$ & 0.0001 \\
\hline BP systolic (mmHg) & $100(90-110)$ & $120(110-130)$ & 0.0001 \\
\hline BP diastolic $(\mathrm{mmHg})$ & $70(60-80)$ & $80(70-90)$ & 0.0002 \\
\hline $\mathrm{HbA}_{1 \mathrm{c}}(\%)$ & $11.9(10.1-14.2)$ & $10.0(8.6-11.8)$ & 0.0002 \\
\hline Cholesterol (mmol/1) & $4.4 \pm 1.3$ & $5.1 \pm 1.4$ & 0.01 \\
\hline Triacylglycerol (mmol/l) & $1.3(0.9-2.2)$ & $1.8(1.4-2.7)$ & 0.003 \\
\hline HDL-cholesterol (mmol/l) & $1.0(0.8-1.2)$ & $0.9(0.8-1.1)$ & NS \\
\hline LDL-cholesterol (mmol/l) & $2.6 \pm 0.9$ & $3.2 \pm 1.3$ & 0.007 \\
\hline C-peptide (pmol/l) & $477(286-845)$ & $788(586-1,136)$ & 0.003 \\
\hline C-peptide-negative, $n(\%)$ & $42(61)$ & $3(8)$ & 0.0001 \\
\hline GADA-positive, $n(\%)$ & $24(35)$ & $5(14)$ & 0.023 \\
\hline
\end{tabular}

insulin, only 11 (29\%) were GADA-positive and 11 (29\%) were C-peptide-positive.

\section{Discussion}

Our study was stimulated by empirical clinical observations by ourselves [8] and others [9, 10, 20] suggesting a remarkably high proportion of apparent type 1 diabetic patients in northern Ethiopia. Accurate epidemiological studies of type 1 diabetes are rare in Africa, but from the data available, results are lower than in western countries. Incidence rates of 1.5 per 100,000 per year have been reported from Tanzania [21] and 10.1 per 100,000 per year from Sudan [22] (differences possibly reflect varying ethnicity of the study populations). A weakness of our study and others $[9-11,13,20]$ is that it was hospital, rather than community-based. However, hospital surveys have been previously used as an argument for the unusually low proportionate rates of type 1 diabetes in sub-Saharan

Table 2 Inter-relations between GADA and C-peptide status in 105 Ethiopian diabetic patients

\begin{tabular}{llll}
\hline Variable & Negative & Positive & $p$ value \\
\hline $\begin{array}{l}\text { C-peptide } \\
\quad n\end{array}$ & 45 & 60 & \\
$\quad$ GADA-positive, $n(\%)$ & $18(40)$ & $11(18)$ & 0.04 \\
$\quad$ On insulin, $n(\%)$ & $42(93)$ & $27(45)$ & 0.0001 \\
GADA & & & \\
$\quad n$ & 29 & 76 & \\
$\quad$ C-peptide-negative, $n(\%)$ & $18(62)$ & $27(35)$ & 0.016 \\
$\quad$ On insulin, $n(\%)$ & $24(83)$ & $45(59)$ & 0.037 \\
\hline
\end{tabular}

Africa, perhaps due to deaths of type 1 patients outside hospital [21, 23]. A strength of our study is that it was conducted in a poor, rural area, whereas most other type 1 research in the continent has been in urban African centres, where nutrition is likely to be at least reasonable [21, 22].

It has long been known that a number of the phenotypic type 1 diabetic patients in northern Ethiopia are very thin, predominantly male [9, 10, 13, 20] and 'frequently discontinued insulin but seldom developed ketoacidosis' [20]. We and others have surmised that because of these clinical observations and the marked past and present undernutrition in the area, these patients may have malnutrition-related diabetes mellitus [9, 13, 24]. The present study measured GADA and IA-2A to internationally accepted specifications [17-19], as well as fasting C-peptide. Although we did not determine glucagon-stimulated C-peptide, there is evidence that fasting $\mathrm{C}$-peptide correlates well with post-stimulation levels $[8,25]$. We also found a clear separation of C-peptide positivity and negativity in our patients.

Our results confirm a previously observed 2:1 male: female ratio and low BMI in rural Ethiopian diabetic patients. Comparison of insulin- and non-insulin-treated patients (Table 1) showed higher age, BMI and BP, and lower $\mathrm{HbA}_{1 \mathrm{c}}$ in the non-insulin treated group, suggestive of type 2 diabetes features. Indeed, BMI in particular showed almost complete separation between the two groups, perhaps supporting differing aetiologies. However, at $66 \%$ the proportion of apparent type 1 diabetes patients remains surprising. Moreover, GADA and C-peptide characteristics by no means always fitted with these categories. Thus, $39 \%$ of the insulin-treated group were C-peptide-positive, and only 35\% were GADA-positive. The failure of C-peptide negativity and GADA positivity to correlate is explored further in Table 2, and led us to subdivide our group into: (1) 'definite' type 1 diabetes (GADA-positive, C-peptide- 
Table 3 Comparison of demographic and biochemical characteristics of definite type 1, definite type 2 and uncertain types of diabetes in northern Ethiopia

\begin{tabular}{|c|c|c|c|c|}
\hline Characteristics & Definite type $1^{\mathrm{a}}$ & Definite type $2^{\mathrm{b}}$ & Uncertain type $^{\mathrm{c}}$ & $p$ value \\
\hline$n(\%)$ & $18(17)$ & $49(47)$ & $38(36)$ & \\
\hline Age (years) & $27(22-38)$ & $53(40-60)$ & $29(22-45)$ & $0.0001^{\mathrm{d}}$ \\
\hline Age at diagnosis (years) & $22(14-29)$ & $43(35-52)$ & $24(16-34)$ & $0.0001^{\mathrm{d}}$ \\
\hline Duration diabetes (years) & $5(4-8)$ & $7(3-13)$ & $5(3-9)$ & $\mathrm{NS}^{\mathrm{e}}$ \\
\hline BMI $\left(\mathrm{kg} / \mathrm{m}^{2}\right)$ & $18.8(17.2-20.1)$ & $23.1(21.1-25.3)$ & $19.2(16.5-20.7)$ & $0.0001^{\mathrm{d}}$ \\
\hline $\mathrm{HbA}_{1 \mathrm{c}}(\%)$ & $12.0(10.0-14.2)$ & $10.2(8.8-12.0)$ & $11.6(10.2-14.2)$ & $0.0113^{\mathrm{f}}$ \\
\hline Insulin treatment, $n(\%)$ & $18(100)$ & $19(38)$ & $34(89)$ & $0.01^{\mathrm{g}}$ \\
\hline GADA-positive, $n(\%)$ & $18(100)$ & $0(0)$ & $11(29)$ & $0.01^{\mathrm{h}}$ \\
\hline C-peptide-negative, $n(\%)$ & $18(100)$ & $0(0)$ & $27(71)$ & $0.01^{\mathrm{i}}$ \\
\hline
\end{tabular}

Data are medians (interquartile range), unless indicated otherwise

Statistical comparison was by Kruskal-Wallis test as non-parametric ANOVA between groups

${ }^{a}$ GADA-positive, C-peptide-negative and on insulin

${ }^{\mathrm{b}}$ GADA-negative, C-peptide-positive and any treatment

${ }^{\mathrm{c}}$ For all comparisons

${ }^{\mathrm{d}}$ For type 2 vs type 1 and uncertain diabetes type; type 1 vs uncertain diabetes type NS

${ }^{\mathrm{e}}$ For all comparisons

${ }^{\mathrm{f}}$ For type 1 vs type 2 diabetes; $p=0.0292$ for type 2 vs uncertain diabetes type; type 1 vs uncertain NS

${ }^{\mathrm{g}}$ For type 1 vs type 2 diabetes; type 1 vs uncertain diabetes type NS

${ }^{\mathrm{h}}$ For type 2 vs uncertain diabetes type; $p=0.0001$ for type 1 vs uncertain diabetes type and for type 1 vs type 2 diabetes

${ }^{\mathrm{i}}$ For type 2 vs uncertain diabetes type and for type 1 vs uncertain type; $p=0.0001$ for type 1 vs type 2 diabetes

negative and on insulin); (2) 'definite' type 2 diabetes (GADA-negative, C-peptide-positive and on any treatment); and (3) 'uncertain' (all other patients) (Table 3). We accept that this categorisation is arbitrary, and that GAD and C-peptide status are not exclusive markers of type 1 and type 2 diabetes. However, although GADA positivity declines after diagnosis of type 1 diabetes, such low rates in a group with median diabetes duration of only 5 years is unusual. In one European study, two-thirds of a type 1 diabetes cohort were still positive for either islet cell antibodies or GADA at 10 years after diagnosis [26]. Also, type 1 diabetic patients elsewhere in Africa, including urban Ethiopia, have been shown to be positive for GADA or islet cell antibodies [27, 28].

Accepting these limitations, our revised groupings give a much more expectable proportion of type 1 diabetes patients at $17 \%$, with type 2 diabetes comprising $47 \%$ and the 'uncertain' group 36\%. Patients in the latter group were closer to type 1 than type 2 diabetes in terms of age (current and at diagnosis), BMI and $\mathrm{HbA}_{1 \mathrm{c}}$, with $89 \%$ on insulin, 29\% GADA-positive and 71\% C-peptide-negative (Table 3). Although this cohort is undoubtedly heterogeneous and may include some GADA-negative type 1 and possibly some GADA-positive type 2 diabetic patients, it seems unlikely that this would account for more than a small proportion. The possibility that these patients may be simply malnourished and poorly controlled type 1 diabetes patients is not supported by their frequency of occurrence or by the variable immunological and C-peptide status. We believe that most of this group represent an unusual form of 'pseudo-type 1' diabetic patients. Interestingly, Siraj and colleagues [8], reporting on C-peptide studies on diabetic patients in Addis Ababa, Ethiopia, commented that 'there is a subgroup of type 2 diabetic subjects with features of type 1 diabetes'.

One distinct possibility is that our insulin-treated patients may have malnutrition-related diabetes [2, 3, 24]. This entity has been described for many decades and is based on the hypothesis that malnutrition in early life leads to pancreatic beta cell damage with varying degrees of insulin hyposecretion. The scenario in northern Ethiopia is certainly compatible with the existence of malnutrition-related diabetes mellitus, since serious famines occurred here in the mid1980s (when most of our insulin-treated patients were children) and food shortages have continued since. Moreover, malnutrition-related diabetes mellitus has been described previously in Ethiopia [29] and, interestingly, a male excess, usually around a 2:1 ratio, is well described in the syndrome $[3,9,24]$. Due to male excess, low BMI, early onset, past (and often present) malnutrition, plus insulin requirement but not dependence, many of our insulin-treated patients fulfil current criteria for malnutrition-related diabetes mellitus. Of 
the two described subgroups of malnutrition-related diabetes mellitus, i.e. fibrocalculous pancreatic diabetes and malnutrition-modulated diabetes mellitus [24], the latter would fit best with our patients. Although we did not perform abdominal $\mathrm{x}$-rays or ultrasounds as part of our research protocol, pancreatic calcification is not seen in the Mekelle area and none of our patients had steatorrhoea. Interestingly, immunological studies on patients with malnutrition-modulated diabetes mellitus in India have shown that many are positive for GADA [30]. In fact, at $23 \%$, the figure is very similar to the $29 \%$ positive rate in our 'uncertain' type group (Table 3). Of further interest, and again similar to our own observations, was that IA-2 positivity was rare. Nevertheless, compelling as these arguments may seem, they are by no means conclusive, and further work is clearly required. One particular problem is that while undernutrition is highly prevalent in northern Ethiopia, the majority of people affected do not develop diabetes.

In conclusion, we believe that we have confirmed an unusual form of 'pseudo-type 1' diabetes from northern Ethiopia, which has clinical and immunological similarities to malnutrition-modulated diabetes mellitus. A wider community-based study of this diabetic population would be valuable, although given the highly scattered population and difficult terrain, such a study would not be easy. More practically, our results may have important implications for insulin treatment in the area and elsewhere. There are often critical shortages of insulin in northern Ethiopia, yet our data show that $40 \%$ of those on insulin treatment were Cpeptide-positive and may therefore be potentially treatable with oral agents, leading to considerable financial savings and helping conserve precious insulin supplies for those who are truly insulin-dependent.

Finally, we agree with Alemu and colleagues that 'the case for malnutrition-related diabetes' should be re-opened [13]. This form of diabetes is no longer recognised by the WHO. We recognise that the observational studies of ourselves and others $[3,9,11,13]$, as well as the immunological and C-peptide data presented here, do not prove causal association. Nevertheless, the clear existence of this unusual type of diabetes in one of the poorest areas of the world makes a strong case for re-evaluating the link between malnutrition and diabetes.

\begin{abstract}
Acknowledgements We are grateful to the patients and staff of the Diabetic Clinic at Mekelle Hospital, Ethiopia. The $\mathrm{HbA}_{1 \mathrm{c}}$ meter was kindly provided by Provalis Diagnostics. A. Tekle was supported by the Tigray Health Bureau. Other funding for travel was provided by an exchange grant from the Association of Physicians of Great Britain and Ireland. Sadly, A. Tekle died following completion of this study. We herewith acknowledge his enormous contribution to clinical medicine and medical research in Mekelle.
\end{abstract}

Duality of interest The authors declare that there is no duality of interest associated with this manuscript.

\section{References}

1. Beran D, Yudkin JS (2006) Diabetes care in sub-Saharan Africa. Lancet 368:1689-1695

2. Gill GV, Mbanya J-C, Ramaiya KL, Tesfaye S (2009) A subSaharan perspective of diabetes. Diabetologia 52:8-16

3. Hart JJ, Tripathy BB (1996) Malnutrition and diabetes in the tropics. Diabetes Care 219:1014-1017

4. Sobngwi E, Mauvais-Jarvis F, Vexiau P, Mbanya J-C, Gautier J-F (2002) Diabetes in Africans. Part 2: ketoacidosis-prone atypical diabetes mellitus. Diabetes Metab 29:5-12

5. Sobngwi E, Choukem SP, Agbalika F et al (2008) Ketosis-prone type 2 diabetes mellitus and human herpesvirus 8 infection in subSaharan Africans. JAMA 299:2770-2776

6. Lester FT (1986) Childhood diabetes mellitus in Ethiopians. Diabet Med 3:278-280

7. Lester FT (1984) The clinical pattern of diabetes mellitus in Ethiopians. Diabetes Care 7:6-11

8. Siraj ES, Reddy SSK, Scherbaum WA, Abdulkadir J, Hammel JP, Faiman C (2002) Basal and post-glucagon C-peptide levels in Ethiopians with diabetes. Diabetes Care 25:453-457

9. Habtu E, Gill G, Tesfaye S (1999) Characteristics of insulinrequiring diabetes in rural northern Ethiopia - a possible link with malnutrition? Ethiopian Med J 37:263-267

10. Brown V, Alemu S, Watkins P (1998) Diabetes in Ethiopia: overcoming the problems of care delivery. J Diabetes Nursing 2:28-31

11. Watkins P, Alemu S (2003) Delivery of diabetes care in rural Ethiopia: an experience from Gondar. Ethiopian Med J 41:9-17

12. Erasmus RT, Blanco-Blanco E, Okesina AB, Matsha T, Gqweta Z, Mesa JA (2001) Prevalence of diabetes mellitus and impaired glucose tolerance in factory workers from Transkei, South Africa. S Afr Med J 91:157-160

13. Alemu S, Dessie A, Seid E et al (2009) Insulin-requiring diabetes in rural Ethiopia: should we reopen the case for malnutritionrelated diabetes? Diabetologia 52:1842-1845

14. World Health Organisation (1999) Definition, diagnosis and classification of diabetes mellitus and its complications. WHO, Geneva

15. Diabetes Control and Complications Trial Research Group (1993) The effect of intensive treatment of diabetes on the development and progression of long-term complications in insulin-dependent diabetes mellitus. New Eng J Med 329:977-986

16. Gebrekidan A, Gill G, Wile D, Gill A, Tesfaye S (2004) An accurate and portable system for glycated haemoglobin measurement in the tropics. Trop Doct 34:94-95

17. Bingley PJ, Bonifacio E, Mueller PW (2003) Diabetes Antibody Standardization Program: first assay proficiency evaluation. Diabetes 52:1128-1136

18. Bingley PJ, Williams AJK (2004) Validation of autoantibody assays in type 1 diabetes - workshop programme. Autoimmunity $37: 257-260$

19. Tom C, Mueller PW, Schlosser M, Bonifacio E, Bingley PJ; Participating Laboratories (2008) Diabetes Antibody Standardisation Program: evaluation of assays for autoantibodies to glutamic acid decarboxylase and islet antigen. Diabetologia 51:846-852

20. Belcher DW (1970) Diabetes mellitus in northern Ethiopia. Ethiopian Med J 8:73-84

21. Swai AB, Lutale JL, McLarty DG (1993) Prospective study of incidence of juvenile diabetes mellitus over 10 years in Dar es Salaam, Tanzania. Brit Med J 306:1570-1572

22. Elamin A, Omer MI, Zein K, Tuvemo T (1992) Epidemiology of type 1 diabetes in Sudan 1987-1990. Diabetes Care 15:1556-1559

23. Motala AA, Omar MAK, Pirie FJ (2000) Type 1 diabetes mellitus in Africa: epidemiology and pathogenesis. Diabetes Int 10:44-47 
24. Abu-Bakare A, Taylor R, Gill GV, Alberti KGMM (1986) Tropical or malnutrition-related diabetes: a real syndrome? Lancet 1:1135-1138

25. Torn C, Landin-Olsson M, Schersten B (2001) Predictability of Cpeptide for auto-immune diabetes in young diabetic patients. Pract Diab Int 18:83-88

26. Savola K, Sabbah E, Kulmala P, Vahasolo P, Honen J, Knip M (1998) Autoantibodies associated with type 1 diabetes mellitus persist after diagnosis in children. Diabetologia 41:1293-1297

27. Panz VR, Kalk WJ, Zouvanis M, Joffe BI (2000) Distribution of autoantibodies to glutamic acid decarboxylase across the spectrum of diabetes mellitus seen in South Africa. Diabet Med 17:524-527
28. Peters WH, Lester FT, Kohnert KD, Hilldman W (1986) The frequency of islet cell surface antibodies in newly diagnosed diabetics from Ethiopia. Exp Clin Endocrinol 87:326-332

29. Abdulkadir J, Mengesha B, Gebriel ZW et al (1990) The clinical and hormonal (C-peptide and glucagon) profile and liability to ketoacidosis during nutritional rehabilitation in Ethiopian patients with malnutrition-related diabetes mellitus. Diabetologia 33:222227

30. Singh AK, Bhatia E, Dabadghao P, Bhatia V, Gellert SA, Colman PG (2000) Role of islet autoimmunity in the aetiology of different clinical subtypes of diabetes mellitus in young north Indians. Diabet Med 17:275-280 